\title{
The cold local Hubble flow as a signature of dark energy
}

\author{
Yu. V. Baryshev ${ }^{1,2}$, A. D. Chernin ${ }^{3,4,5}$, and P. Teerikorpi ${ }^{3}$ \\ 1 Institute of Astronomy, St. Petersburg State University, Staryj Peterhoff, 198504 St. Petersburg, Russia \\ ${ }^{2}$ Isaac Newton Institute of Chile, Saint-Petersburg Branch, Russia \\ 3 Tuorla Observatory, University of Turku, 21500 Piikkiö, Finland \\ 4 Division of Astronomy, University of Oulu, 90014 Finland \\ 5 Sternberg Astronomical Institute, Moscow University, 119899 Moscow, Russia
}

Received 9 May 2001 / Accepted 6 September 2001

\begin{abstract}
The Local Group environment at 1-10 Mpc expands linearly and smoothly, as if ruled by uniform matter, while observations show on the same scales the very lumpy local galaxy universe. This enigma in cosmology has also been demonstrated by high-resolution $N$-body CDM simulations. We suggest that the homogeneous dark energy component, revealed by SNIa observations, may resolve the problem of the local cold Hubble flow within the highly non-uniform environment. Linear density perturbations on a homogeneous background with the equation of state $p_{\mathrm{Q}}=w \rho_{\mathrm{Q}} c^{2}$ are decaying for $w<-1 / 3$. Exact non-linear Einstein's equations for a spherically symmetric matter concentration, show that there is a zero-mass surface where the positive mass of the local cloud is compensated by the negative dark energy mass, and beyond this surface dark energy dominates dynamically. In such regions the velocity dispersion is adiabatically cooling, and this may explain why the Hubble law starts on the outskirts of the Local Group, with the same $H_{0}$ as globally and with a remarkably small velocity dispersion.
\end{abstract}

Key words. dark matter - cosmological parameters - Local Group

\section{Introduction}

Recent studies of the local volume $(<10 \mathrm{Mpc})$ show that the velocity dispersion around the local Hubble law is very low $\left(\sigma_{\mathrm{V}}<40 \mathrm{~km} \mathrm{~s}^{-1}\right)$ (Ekholm et al. 2001; Karachentsev \& Makarov 2000). This highlights the riddle of the local Hubble law, first recognized by Sandage et al. (1972): Why is the Hubble flow so linear and cold in the very lumpy environment of the Local Group? The $N$-body CDM simulations by Governato et al. (1997) have fully confirmed and even sharpened this problem.

Here we argue that a possible solution for this problem may be found in the remarkable new coincidence that the antigravity of dark energy starts to dominate over the gravity of lumpy matter at the distance where the Hubble flow emerges.

\section{The enigma of the local Hubble flow}

\subsection{Formulation of the problem}

In the standard cosmology the Hubble law, $V=H \times r$, is a strict consequence of a uniform distribution of selfgravitating matter (Robertson 1955; Peebles 1993). Hence

Send offprint requests to: P. Teerikorpi, e-mail: pekkatee@astro.utu.fi the predicted linear velocity field is only valid for scales where the universe is uniform. However, a very puzzling fact long ago noted by Sandage et al. (1972), is that Hubble discovered his law in the distance interval 1$20 \mathrm{Mpc}$, where the galaxies are very clumpily distributed. Indeed, this was deep inside a cell of uniformity having the size of at least $20 \mathrm{Mpc}$, which follows from the powerlaw correlation function for field galaxies (Peebles 1993). On all smaller scales the galaxies are fractally distributed, forming strongly inhomogeneous structures (Sylos Labini et al. 1998; Wu et al. 1999; Tikhonov et al. 2000). There is also observational evidence that fractality continues up to 100-200 Mpc (de Vaucouleurs 1971; Sylos Labini et al. 1998; Teerikorpi et al. 1998).

On the other hand, in the same local volume observations reveal a linear distance-velocity relation which starts from the distance of about $1 \mathrm{Mpc}$ and shows extremely low velocity scatter with a dispersion $<40 \mathrm{~km} \mathrm{~s}^{-1}$ (Ekholm et al. 2001; Karachentsev \& Makarov 2000). The linear Hubble law continues with the same Hubble constant also outside the local volume up to larger distances (Teerikorpi 1997; Ekholm et al. 1999; Giovanelli et al. 1999).

In the highly nonuniform local universe such a cold linear Hubble flow seems to contradict the standard picture where the Hubble law is the strict consequence of homogeneity of matter distribution. As illustrated by 
Weinberg's words: "in fact, we would not expect any neat relation of proportionality between velocity and distance for these [closeby] ... galaxies" (Weinberg 1977). A quantitative formulation and study of this paradox was given by Baryshev et al. (1998). Sandage (1999, also 1986) recently expressed this surprising situation, as the "extremely local rate [of expansion] is the same as the global rate to better than $10 \%$ ", and an "explanation of why the local expansion field is so noiseless remains a mystery".

\subsection{The Local Group as a test of CDM models}

The expected coldness of the local Hubble flow was studied quantitatively by Governato et al. (1997) using high resolution CDM $N$-body simulations. They identified many "Local Groups" to match the observed dynamics and environments of our Local Group. Calculation of velocity dispersions in $5 \mathrm{Mpc}$ volume around the "LG's" led to an important result. For $\Omega=1 \mathrm{CDM}$ model the peculiar velocities have $300 \mathrm{kms}^{-1}<\sigma_{\mathrm{V}}<700 \mathrm{kms}^{-1}$, and for $\Omega=0.3$ CDM model $150 \mathrm{~km} \mathrm{~s}^{-1}<\sigma_{\mathrm{V}}<300 \mathrm{kms}^{-1}$. They state that "neither of these models are able to produce a single LG with a local velocity dispersion comparable with the observed value". Also, no biasing scheme could reproduce the cold local flow.

Karachentsev (1996) concluded that the Local Group is typical among nearby groups. Governato et al. (1997) also found that the LG candidates occupy typical places in the hierarchic CDM structures. This justifies the use of the environment of our Local Group as a representative test object.

One may thus divide the enigma of the local Hubble flow into the following problems:

(1) The linear velocity law appears at a distance 1.5 Mpc, a small fraction of the scale where the galaxy universe may finally appear uniform. Why does our highly non-uniform environment expand as if it were uniform?

(2) What makes the local Hubble flow so cold? Not only does the Hubble law exist, but it has a remarkably small scatter $\left(\sigma_{\mathrm{V}} \leq 40 \mathrm{~km} \mathrm{~s}^{-1}\right)$.

\section{Dark energy and dynamical model}

\subsection{Vacuum, quintessence, dark energy}

The Hubble diagram for high redshift type Ia Supernovae (Riess et al. 1998; Perlmutter et al. 1999) and the Boomerang and MAXIMA-1 measurements of the first acoustic peak location in the angular power spectrum of the CMB (de Bernardis et al. 2000; Jaffe et al. 2000) restrict the standard cosmological model close to the critical density $\left(\Omega=\Omega_{\mathrm{m}}+\Omega_{\Lambda}=1\right)$, with a dominant $\Lambda$-like, "dark energy" (DE) component at the present epoch $\left(\Omega_{\Lambda}^{0} \approx 0.7\right)$.

The detection of the DE component provides a natural candidate for a high-density uniform background. In the cosmological theory there are many kinds of dark energy candidates with positive energy density $\left(\rho_{\mathrm{Q}} c^{2}>0\right)$ and negative pressure $\left(p_{\mathrm{Q}}<0\right)$. The dark energy is a common name for: Einstein's cosmological constant $\Lambda$ or cosmological vacuum, time-variable $\Lambda$, scalar fields, and exotic substances with negative pressure (Bahcall et al. 1999; Sahni \& Starobinsky 2000). It is now much studied and also included in cosmological tests (Wang et al. 2000; Podariu \& Ratra 2000). The main motivation for evolving DE is the "cosmic coincidence": Why is the rapidly decreasing energy density of matter just now close to that of the unchanging vacuum?

A striking property of the DE having the equation of state $p_{\mathrm{Q}}=w \epsilon_{\mathrm{Q}}, w \in[-1,0)$, is that its gravitating mass

$M_{\mathrm{Q}}=\frac{4 \pi}{3}(1+3 w) \rho_{\mathrm{Q}} r^{3}$

is negative for $w<-1 / 3$. This produces cosmological antigravity which accelerates the expansion of the universe. E.g. for Einstein's $\Lambda=8 \pi G \rho_{\Lambda} / c^{2}$ (cosmological vacuum with $w=-1) M_{\Lambda}=-8 \pi \rho_{\Lambda} r^{3} / 3$.

An important class of DE models is the so-called coupled quintessence (see e.g. Wetterich 1995; Amendola 1999), where the total (matter + DE) energy momentum tensor is conserved, in contrast to ordinary quintessence where the matter and DE are separately conserved. For coupled quintessence, a stationary DE model was recently found (Amendola \& Tocchini-Valentini 2000) which predicts coherent behaviour of matter and DE density at late cosmic epochs. Below we show that especially such models are favorable for explaining the cold local Hubble law.

\subsection{Zero-mass radius for spherical inhomogeneity}

We study a model where a separate spherical matter "cloud" lies on a homogeneous DE background. The dark energy exists alongside with luminous and dark matter in the immediate environment of the matter cloud. Cosmological vacuum $(w=-1)$ is truly uniform. Quintessence in general may vary in time and space, but in some models DE may be regarded as homogeneous on scales up to $\approx 100 \mathrm{Mpc}$ (Wang \& Steinhardt 1998). The dynamics of the cloud is determined by the competition of the gravity of matter and the antigravity of the DE.

Now consider the radial law of the matter density. The mass-to-luminosity ratio for different galaxy systems seems to remain constant for $r>0.5 \mathrm{Mpc}$ (Bahcall et al. 1995), hence dark matter is distributed like luminous matter on such scales. The mean density of luminous (and hence, of dark) matter decreases with increasing scale so that the mass grows as $M_{\mathrm{m}}(r) \propto r^{D}$, where $D$, the fractal dimension, is between 1 and 3 for scales at least up to 20 Mpc (Sylos Labini et al. 1998; Wu et al. 1999; Tikhonov et al. 2000). Further evidence for the power-law density comes from number counts of galaxies. It was shown by Baryshev (1981), Klypin et al. (1989), and Teerikorpi et al. (1998) that the galaxy density around our Galaxy behaves as a power-law with the exponent corresponding to $D \approx 2$ up to scales of about $200 \mathrm{Mpc}$.

A recent analysis of the correlation function for a sample of 330 galaxies within the local volume $(<10 \mathrm{Mpc})$ 
showed a fractal-like galaxy distribution with dimension $D=1.8$ (Tikhonov et al. 2000). This also justifies the power law as a description of galaxy distribution around the LG.

The density of the homogeneous DE component is constant $\left(M_{\mathrm{Q}}(r) \propto r^{3}\right)$, hence it starts to dominate the matter component after a distance $r_{\mathrm{Q}}$. To estimate $r_{\mathrm{Q}}$ for the environment of the Local Group, we use two models. The first model generalizes Sandage's (1986) point-mass model: a mass $M$ is placed on the dark energy background with density $\rho_{\mathrm{Q}}$. In the second model, a spherical mass distribution $M_{\mathrm{m}}(r)$ is on the DE background.

The dynamics of a spherically symmetric dust matter cloud with density $\rho_{\mathrm{m}}(r)$ and energy-momentum tensor $T_{(\mathrm{m})}^{i k}=\rho_{\mathrm{m}}(r) c^{2} \operatorname{diag}(1,0,0,0)$ on the homogeneous quintessence background having $T_{(\mathrm{Q})}^{i k}=$ $\rho_{\mathrm{Q}} c^{2} \operatorname{diag}(1, w, w, w)$ is described by the exact Eintein's field equations. The $(1,1)$ component of the field equation, in combination with the $(0,0)$ component, gives the following exact equation of motion:

$\ddot{r}=-G M_{\mathrm{eff}} / r^{2} ; \quad M_{\mathrm{eff}}=M_{\mathrm{m}}(r)+M_{\mathrm{Q}}(r)$.

Here $r(t, \chi)$ is the radial distance from the centre of the spherical cloud, $M_{\mathrm{m}}(r)=4 \pi \int_{0}^{r} \rho_{\mathrm{m}}(r) r^{2} \mathrm{~d} r$ is the dust mass within the sphere of radius $r, M_{\mathrm{Q}}(r)$ is the quintessence mass within the same radius and is given by Eq. (1).

For the point-mass model $M_{\mathrm{m}}(r)=M$ there is a distance $r_{\mathrm{Q}}$ where $\ddot{r}=0$ and the DE mass energy equals that of the matter cloud, i.e. $M_{\text {eff }}=0$. This zero-mass radius is:

$r_{\mathrm{Q}}=\left(3 M /\left(4 \pi \tilde{w} \rho_{\mathrm{Q}}\right)\right)^{1 / 3}$

where $\tilde{w}=-3 w-1$. For $w=-1 / 3$ the gravitating mass of the DE is zero and formally $r_{\mathrm{Q}} \rightarrow \infty$.

In the second model $\left(M_{\mathrm{m}}(r)=M_{*}\left(r / r_{*}\right)^{D}\right)$ the dark energy term dominates dynamically at distances

$r>r_{\mathrm{Q}}=\left(r_{*}\right)^{-\frac{D}{3-D}}\left(M_{*}\right)^{\frac{1}{3-D}}\left(3 / 4 \pi \tilde{w} \rho_{\mathrm{Q}}\right)^{\frac{1}{3-D}}$.

Note that the distance $r_{\mathrm{Q}}$ is defined by the ratio between the local cloud mass and the global density of quintessence. Beyond the zero-mass (screening) distance $r_{\mathrm{Q}}$ dark energy density dominates over the matter density. The zero-mass distance for a given mass cloud depends on cosmic time $t$ (or scale factor $a$ ).

\subsection{The temporal behaviour of $r_{Q}$ and critical time $t_{Q}$}

When the uniform DE background expands, a galaxy which now is on the border of the DE dominated region near the matter cloud, previously lied inside the gravity dominated sphere. In terms of comoving coordinates, the border $r_{\mathrm{Q}}$ shifts outwards for increasing redshift. We calculate the temporal behaviour of the screening distance for a few kinds of density evolution. The relation between density and scale factor for a substance with the equation of state $p=w \rho c^{2}$ is

$\rho \propto a^{-3(1+w)}$ where $a=S(t) / S\left(t_{0}\right)$ is the normalized scale factor and $t_{0}$ is the present epoch. We consider such epochs when the average (homogeneous) density of matter component $\rho_{\mathrm{mh}}$ is less than the DE density $\rho_{\mathrm{Q}}$, as suggested by observations.

To illustrate the model dependence of the zero-mass distance we also take a model of "coherent" behaviour for matter and quintessence: $\rho_{\mathrm{Q}}=k \rho_{\mathrm{mh}}$, where $\rho_{\mathrm{mh}}$ is the average matter density. Such a relation was studied by Baryshev et al. (2000) in order to solve the problem of the low velocity dispersion in the local Hubble flow. It naturally appears at the late cosmic epoch within the model of stationary coupled dark energy, recently found by Amendola \& Tocchini-Valentini (2000). For the coherent phase of the DE there is a simple relation between the density and the scale factor. The condition that the covariant divergence of the total energy-momentum tensor $(\mathrm{DE}+$ matter $)$ is zero implies that $\dot{\rho}=-3\left(\rho+\frac{p}{c^{2}}\right) \dot{a} / a$, where $\rho=\rho_{\mathrm{Q}}+\rho_{\mathrm{mh}}$ and $p=p_{\mathrm{Q}}$ for dust-like matter $\left(p_{\mathrm{m}}=0\right)$. Hence the coherent DE density behaves as

$\rho_{\mathrm{Q}} \propto a^{-3\left(\frac{1+k+w k}{1+k}\right)}$.

For $\frac{1}{k}=0$ (no uniform matter) Eq. (6) gives the usual quintessence behaviour Eq. (5). General properties of 2-fluid FLRW models (including coherent matter \& DE) are studied elsewhere (Baryshev et al. 2001).

As an example we calculate the shift of $r_{\mathrm{Q}}$ in time for three DE models: 1) Cosmological constant $\Lambda$, 2) quintessence with $w=-2 / 3$, and 3) coherent quintessence with $w=-2 / 3, k=1$. First, we consider the ratio between two metric distances $r_{\text {gal }} / r_{\mathrm{Q}}$, where $r_{\text {gal }}$ is the distance to a galaxy which now is in the DE dominated region and takes part in the Hubble flow. The distance $r_{\text {gal }}$ is simply proportional to $a$. The behavior of the critical distance $r_{\mathrm{Q}}$ depends on the DE and matter models. E.g. for the point-mass model the ratio is $r_{\text {gal }} / r_{\mathrm{Q}}=a^{(-w /(1+1 / k))}$, which for $1 / k=0$ gives $a^{-w}$, corresponding either to Einstein's $\Lambda(w=-1)$ or to the quintessence $(w \leq-1 / 3)$.

We define a characteristic time when the antigravity dominates in the vicinity of a matter cloud. It is counted from the local critical epoch $t_{\mathrm{Q}}$, corresponding to the scale factor $a_{\mathrm{Q}}=a\left(t=t_{\mathrm{Q}}\right)$ when DE antigravity starts to exceed the gravity of the matter cloud for a galaxy which presently $\left(t=t_{0}\right)$ is at the distance $r_{\text {gal }}=2 r_{\mathrm{Q}}^{0}$, well within the DE dominated region. The exact value of the numerical factor is arbitrary, but for the LG the number two corresponds to $r_{\text {gal }} \approx 4 \mathrm{Mpc}$, i.e. intergroup distances. E.g. for Sandage's point-mass model thus defined critical scale factor is

$a_{\mathrm{Q}}=2^{(1+1 / k) / w}$.

With $1 / k=0$ this gives $a_{\mathrm{Q}}=0.5$ for $w=-1$ and $a_{\mathrm{Q}}=$ 0.354 for $w=-2 / 3$, while with $k=1, w=-2 / 3$ the critical epoch was earlier at $a_{\mathrm{Q}}=0.125$, and the galaxy spends a longer time in the DE dominated region. Note that the local critical time $t_{\mathrm{Q}}$ differs from the global time when DE starts dominating over average matter. 


\section{Structure growth and peculiar velocities in DE dominated regions}

The effect of $\Lambda$ on the evolution of primordial density fluctuations was studied by Peebles (1980, 1984), Lahav et al. (1991), Carroll et al. (1992). Matter perturbations in quintessence models are discussed by Wang \& Steinhardt (1998), Ma et al. (1999), Sahni \& Starobinsky (2000), and Fabris \& Goncalves (2000). It was shown that even for $\Omega_{\mathrm{Q}}^{0} \sim 1$ the DE has only a slight effect on the total growth factor and the main effect comes from $\Omega_{\mathrm{m}}^{0}$. The small influence derives from the short time interval when DE dominates during the late epoch of structure formation. These considerations of the growth factor have led to the claim that the dark energy component can not produce observable effects in the local dynamics.

However, we emphasize that there are several reasons why the DE component could have observable influence on the structure formation dynamics. First, in different regions of the universe, DE has dominated vastly different time intervals, depending on the local matter density contrast. Second, according to the exact dynamical equation (Eq. (2)), on scales where cosmological antigravity overcomes the gravity of local matter concentration, the dynamics of a test galaxy does not depend on this local mass concentration and hence undergoes adiabatic cooling. Third, the existence of the growing mode itself essentially depends on the equation of state of the background fluid. Indeed, let us consider the linear growth of a density perturbation on a homogeneous background with the equation of state $p_{\mathrm{Q}}=w \rho_{\mathrm{Q}} c^{2}$ (constant $w$ ). As is well-known (Heath 1977; Peebles 1980; Eisenstein 1997; Peacock 1999) for the density perturbation $\delta=\delta \rho_{\mathrm{m}} / \rho_{\mathrm{mh}}=\left(\rho_{\mathrm{m}}-\rho_{\mathrm{mh}}\right) / \rho_{\mathrm{mh}}$, where $\rho_{\mathrm{m}}$ and $\rho_{\mathrm{mh}}$ are the matter density and the average (uniform) background matter density, there are two modes: decaying, where $\delta_{1} \propto \dot{a} / a$, and growing:

$\delta_{2} \propto \frac{\dot{a}}{a} \int^{a}(\dot{a})^{-3} \mathrm{~d} a$

For the flat FLRW model, $\dot{a} / a=H(a) \propto a^{-\frac{3(1+w)}{2}}$, and

$\delta_{2} \propto a^{(1+3 w)}$.

Hence for $\mathrm{DE}(w<-1 / 3)$ the mode $\delta_{2}$ is decaying, too.

Therefore in the regions of the universe where dark energy dominates new structures do not condense and linear perturbations of density and peculiar velocities decay. This effect was considered for vacuum-dominated regions by Chernin et al. (2000) using Zeldovich's (1965) analysis of gravitational instability.

The zero-mass radius $r_{\mathrm{Q}}$ gives the position of the border between the matter and DE dominated regions. If a galaxy now beyond $r=r_{\mathrm{Q}}$ also in the past was long enough in the DE dominated region, then the peculiar velocities induced by local masses have adiabatically cooled down $(\delta V($ now $)=\delta V(z) a(z))$. Such DE dominated regions between mass clouds are "pacific oceans" where the linear Hubble law appears and the global $H_{0}$ may be measured even locally.

\section{Possible explanation of the remarkable properties of the local Hubble flow}

\subsection{The zero-mass radius $r_{\mathrm{Q}}$ for the Local Group}

Let us estimate the distance $r_{\mathrm{Q}}$ for the Local Group in the case of the point-mass model and the cosmological vacuum ( $w=-1$ and $\rho_{\mathrm{Q}}=\rho_{\Lambda}$ is constant in time). Van den Bergh (1999) gives the mass of the LG as $M_{\mathrm{LG}}=2 \times 10^{12} M_{\odot}$. We adopt this value but note that in recent data there is a tendency for the LG mass to decrease (Evans et al. 2000; Karachetsev \& Makarov 2000).

The vacuum density from SNIa observations is $\rho_{\Lambda} \simeq$ $0.7 \rho_{\text {crit }}=4.7 \times 10^{-30} h_{60}^{2} \mathrm{~g} / \mathrm{cm}^{3}$. Then the distance $r_{\Lambda}$, where the vacuum starts to dominate, is $\simeq 1.5 \mathrm{Mpc}$ in the point-mass model. If one changes $M_{\mathrm{LG}}$ or $\rho_{\Lambda}$ by a factor of two, the zero-mass border shifts by 26 percent. If one chooses the mass model with $D=1$ and $M_{*}=2 \times 10^{12} M_{\odot}$ at $r_{*}=1 \mathrm{Mpc}$, Eq. (4) yields $r_{\Lambda}=1.8 \mathrm{Mpc}$.

Thus $r_{\Lambda}$ is robustly put into the range 1 to $2 \mathrm{Mpc}$. This value is surprisingly close to the distance where the Hubble law emerges, or 1.5 Mpc (Sandage 1986). Is this just a coincidence? We think that this is a key feature of the local matter flow. The dominance of the dark energy at 1-2 Mpc and beyond means that it provides the uniform background for the Hubble flow of matter on such scales.

\subsection{Zero-velocity surface and the visibility of the local DE region}

For predicting the distance where the growing structure separates from the Hubble flow we use the relation between peculiar velocity and density fluctuation in the linear gravitational growth theory with $\Lambda$ (Peebles 1984):

$$
\frac{\delta v(r)}{v_{\mathrm{H}}}=\frac{1}{3} \Omega_{0 \mathrm{~m}}^{0.6} \frac{\delta \rho(r)}{\rho_{\mathrm{mh}}}
$$

where $\delta v(r)$ is the peculiar velocity, $v_{\mathrm{H}}=H_{0} r$ is the Hubble velocity, $\Omega_{0 \mathrm{~m}}$ is the present matter density parameter. An exact nonlinear spherical model of the velocity flow within a power-law density field was recently studied by Gromov et al. (2001) who confirmed the use of Eq. (10) as a first approximation also for the vacuum. In the general case of DE the validity of this relation should be studied separately, though the studies on the growth factor (Wang \& Steinhardt 1998; Ma et al. 1999) suggest that it may be used for any $w$.

In our model of the LG the density fluctuation is $\delta \rho(r)=\rho_{\mathrm{m}}(r)-\rho_{\mathrm{mh}}=\rho_{\mathrm{mh}}\left(r / r_{0}\right)^{-\gamma}$, with $\gamma=3-D$. From the condition $\delta v\left(r_{\mathrm{zV}}\right) \approx v_{\mathrm{H}}$ we get the zero-velocity distance $r_{\mathrm{zv}}$ in terms of the LG mass $M_{\mathrm{LG}}$ within $1 \mathrm{Mpc}$ :

$r_{\mathrm{zv}}=\left(\frac{0.53 D}{\Omega_{0 \mathrm{~m}}^{0.4}}\right)^{1 / \gamma}\left(\frac{M_{\mathrm{LG}}}{2 \times 10^{12} M_{\odot}}\right)^{1 / \gamma}$. 
For $D=1$ and $\Omega_{0 \mathrm{~m}}=0.3$ we get $r_{\mathrm{zv}}=0.93 \mathrm{Mpc}$, and for $D=2, r_{\mathrm{zv}}=1.7 \mathrm{Mpc}$. Hence if the mass of the $\mathrm{LG}$ is about $2 \times 10^{12} M_{\odot}$, in this case the zero-mass distance $r_{\mathrm{Q}}$ is close to the zero-velocity distance $r_{\mathrm{zv}}$, and this allows us to see the Hubble flow within the local DE dominated region.

\subsection{The local and global Hubble constant}

As the short zero-velocity distance for the LG allows one to see the DE dominated region, characterized by the cold linear Hubble flow, one expects that the local value of the Hubble ratio is not far from the global Hubble constant. Indeed, observations confirm this on a few percent level (Teerikorpi 1997; Ekholm et al. 1999; Giovanelli et al. 1999; Freedman et al. 2001). Even with the recently suspected significant bias in extragalactic cepheid distances (Teerikorpi \& Paturel 2001), this agreement between the local and global rates of expansion remains.

Above we calculated the DE density from $\Omega_{\Lambda}$ (from SNIa) and assuming $h=0.6$. By the way, if we had, independently of the Hubble constant, some local measurement of the dominant uniform DE density, we could test the consistency of the adopted cosmological model by predicting the value of the Hubble constant via the relation $H_{0} \approx\left(8 \pi G \rho_{\mathrm{Q}} / 3\right)^{1 / 2}$ for the $\Omega=1$ universe. For example, if we roughly identify the distance $r_{\text {start }}$ where the Hubble law starts, with the screening radius $r_{\mathrm{Q}}$ for our Local Group, then we can calculate $\rho_{\mathrm{Q}}$ from Eq. (3) and the expected value of $H_{0}$ is

$H($ predicted $) \approx\left(2 G M_{\mathrm{LG}} / \tilde{w}\right)^{1 / 2}\left(r_{\text {start }}\right)^{-3 / 2}$

where $M_{\mathrm{LG}}$ is the mass of the Local Group. With the "standard" values $M=2 \times 10^{12} M_{\odot}$ and $r_{\text {start }}=1.5 \mathrm{Mpc}$, the Eq. (12) gives for $H_{0}$ values from $53 \mathrm{~km} \mathrm{~s}^{-1} / \mathrm{Mpc}(w=$ $-1)$ to $74 \mathrm{~km} \mathrm{~s}^{-1} / \mathrm{Mpc}(w=-2 / 3)$, covering the values obtained for $H_{0}$ by different methods.

\subsection{The coldness of the local Hubble flow}

The peculiar velocities in the vicinity of the Local Group are produced by all nearby mass inhomogeneities. Because the distance $r_{\mathrm{Q}}$ for the LG is less than the characteristic distance between similar groups, one can consider the galaxies beyond $r_{\mathrm{Q}}$ (but in the intergroup space) as adiabatically cooling free particles.

The possibility to solve the problem of low velocity dispersion may be illustrated by the following simplified reasoning. At any epoch $a(t)$ and on the scale $r$ one may regard $\delta V \approx H r$ as an upper limit to the scatter around the Hubble law. In the non-linear clustering regime the velocity dispersion is about $\mathrm{Hr}$ for a cluster with size $r$ (Peebles 1980). In the linear regime, $\delta v(r) / H r \sim \delta \rho(r) / \rho_{\mathrm{mh}}$ leads to $\delta v<H r$.

For models with zero curvature the Hubble constant $H(a) \propto a^{-(3 / 2) \beta}$, where $\beta=(1+k+w k) /(1+k)$. E.g. in the case of vacuum, i.e. $w=-1$ and $1 / k=0$ we have $H=$ const., and for dust matter $k=0$ hence $H \propto a^{-3 / 2}$.
So the scatter $\delta V(a)<H_{0} r_{0} a^{1-(3 / 2) \beta}$, where $r_{0}$ is the considered scale at the present epoch. E.g. for $r_{0}=5 \mathrm{Mpc}$ and $H_{0}=60 \mathrm{~km} \mathrm{~s}^{-1} / \mathrm{Mpc}, \delta V(a)<300 a^{1-(3 / 2) \beta} \mathrm{km} \mathrm{s}^{-1}$. This is also valid at the critical epoch $a_{\mathrm{Q}}$ after which the peculiar velocities within a DE dominated region start to cool down adiabatically, so that $\delta V($ now $)=\delta V\left(a_{\mathrm{Q}}\right) a_{\mathrm{Q}}$. Hence one expects now $\delta V$ (now) $<300 a_{\mathrm{Q}}^{2-(3 / 2) \beta} \mathrm{km} \mathrm{s}^{-1}$. More conservatively, we also calculate a (larger) upper limit for $\delta V$ (now) from the relation for dust $(\beta=1)$, noting that actually the dark energy does not fully dominate all the way from $a=a_{\mathrm{Q}}$ to $a=1$.

For examples we use the epoch $a_{\mathrm{Q}}$ from Sect. 3.3. In the case of the cosmological constant, $a_{\mathrm{Q}}=0.500$, hence $\delta V$ (now $)<75-212 \mathrm{~km} \mathrm{~s}^{-1}$. For the DE with $w=-2 / 3$, $a_{\mathrm{Q}}=0.354$ and $\delta V$ (now) $<63-178 \mathrm{~km} \mathrm{~s}^{-1}$, and for the coherent evolution $w=-2 / 3, k=1$, the critical $a_{\mathrm{Q}}=$ 0.125 and one expects $\delta V$ (now) $<38-106 \mathrm{~km} \mathrm{~s}^{-1}$.

The numbers used above are not a unique choice, but they well illustrate the expected cooling of the velocity dispersion in different DE models. A complete treatment of the dispersion problem needs high-resolution large-volume simulations of structure formation on the evolving DE background, taking into account the cooling processes in the dark energy dominated regions.

\section{Discussion and conclusions}

Cosmological dark energy is now recognized as an important constituent of the universe (see reviews by Bahcall et al. 1999; Sahni \& Starobinsky 2000; Chernin 2001). It gives a new possibility to resolve the old puzzle of the linear Hubble law existing within the highly nonuniform local galaxy universe. The antigravity and homogeneity of the cosmological DE may explain the quiet Hubble flow close to the Local Group.

In dark energy dominated regions of the universe ("pacific oceans") the Hubble law exists due to the uniformity of the DE component, with the Hubble constant determined by the dark energy density. The coldness of the local Hubble flow follows from the fact that a galaxy, spending a sufficient time in the DE dominated region where linear perturbations decay as $\delta \propto a^{(1+3 w)}$, looses its peculiar velocity via adiabatic cooling. Hence the mysterious quietness of the local Hubble flow does not look dramatic in the context of the DE driven expansion. The most effective decrease of velocity dispersion is found for stationary coupled quintessence (Amendola \& Tocchini-Valentini 2000; Baryshev et al. 2000) where the DE density changes coherently with the matter density in the late cosmic epoch.

High-resolution $N$-body simulations for QCDM models could in principle restrict the evolution of dark energy in late cosmological epochs, from an analysis of the velocity scatter around "LG" candidates. The existing $N$-body results for $\Lambda$ CDM models (Jenkins et al. 1998) give similar velocity dispersions as OCDM models (Governato et al. 1997), much higher than observed around the Local Group. In comparison, analytical results, based on the exact dynamical equation (Eq. (2)) which is the $(1,1)$ 
component of Einstein's equations, show that there is a zero-mass surface for any spherical matter concentration, beyond which the dark energy dominates. In such regions velocity dispersion is adiabatically cooling down. This underlines the question how to take into account the analytical results in $N$-body simulation methods.

We conclude that even the closeby universe is now seen as a cosmic laboratory where all physical ingredients: luminous matter, dark matter, and dark energy, may be detected. Cosmology starts immediately beyond the border of the Local Group. In other environments the DE dominance starts at smaller or larger distances. So, around the Coma cluster the critical distance is $\approx 20 \mathrm{Mpc}$. For a cosmologist in Coma, cosmology begins around such a distance.

Acknowledgements. We are grateful to Chris Flynn for helpful comments and to the referees L. Amendola and F. Governato for useful criticism and suggestions. Yu. B. and P. T. remember with pleasure the hospitality by Georges Paturel, when they attended the informal Lyon meeting on cosmology in April 2000. During this visit Yu. B. and P. T. performed the part of this work concerning the coherently behaving matter and dark energy. This study has been supported by The Academy of Finland (projects "Cosmology from the local to the deep galaxy universe" and "Galaxy streams and dark matter structures").

\section{References}

Amendola, L. 1999 [astro-ph/9908023]

Amendola, L., \& Tocchini-Valentini, D. 2000 [astro-ph/0011243]

Bahcall, N. A., Lubin, L. M., \& Dorman, V. 1995, ApJ, 447, L81

Bahcall, N., Ostriker, J., Perlmutter, S., \& Steinhardt, P. 1999, Science, 284, 1481

Baryshev, Yu. 1981, Izvestiya SAO, 14, 24

Baryshev, Yu., Gromov, A., \& Teerikorpi, P. 2001, in prep.

Baryshev, Yu., Sylos Labini, F., Montuori, M., Pietronero, L., \& Teerikorpi, P. 1998, Fractals, 6, 231

Baryshev, Yu., Chernin, A., \& Teerikorpi, P. 2000 [astro-ph/0011528]

Carroll, S., Press, W., \& Turner, E. 1992, ARA\&A, 30, 499

Chernin, A. 2001, Physics-Uspekhi, 44, in press

Chernin, A., Teerikorpi, P., \& Baryshev, Yu. 2000, Adv. Space Res., in press [astro-ph/0012021]

Davis, M., \& Peebles, P. J. E. 1983, ApJ, 267, 465

de Bernardis, P., Ade, P. A. R., Bock, J. J., et al. 2000, Nature, 404, 955

de Vaucouleurs, G. 1971, Science, 167, 1203

Eisenstein, D. 1997 [astro-ph/9709054]

Ekholm, T., Baryshev, Yu., Teerikorpi, P., Hanski, M., \& Paturel, G. 2001, A\&A, 368, L17

Ekholm, T., Lanoix, P., Teerikorpi, P., Paturel, G., \& Fouqué, P. 1999, A\&A, 351, 827
Evans, N., Wilkinson, M., Guhathakurta, P., Grebel, E., \& Vogt, S. 2000, ApJ, 540, L9

Fabris, J. C., \& Goncalves, S. V. B. 2000 [gr-gc/0010046]

Freedman, W. L., Madore, B., Gibson, B. K., et al. 2001, ApJ, 553,47

Giovanelli, R., Dale, D., Haynes, M., Hardy, E., \& Campusano, L. 1999, ApJ, 525, 25

Governato, F., Moore, B., Cen, R., et al. 1997, New Astr., 2, 91

Gromov, A., Baryshev, Yu., Suson, D., \& Teerikorpi, P. 2001, Grav. \& Cosm., 7, No. 2(26), 140

Heath, D. 1977, MNRAS, 179, 351

Jaffe, A. H., Ade, P. A. R., Balbi, A., et al. 2000, Phys. Rev. Lett. 86, 3475

Jenkins, A., Frenk, C., Pearce, F., et al. 1998, ApJ, 499, 20

Karachentsev, I. 1996, A\&A, 305, 33

Karachentsev, I., \& Makarov, D. 2000, Preprint 151, Russian Academy of Science

Klypin, A., Einasto, L., Einasto, M., \& Saar, E. 1989, MNRAS, 237, 929

Lahav, O., Lilje, P., Primack, J., \& Rees, M. 1991, MNRAS, 251, 126

Ma, C., Caldwell, R., Bode, P., \& Wang, L. 1999, ApJ, 521, L1

Peacock, J. A. 1999, Cosmological Physics (Cambridge: Cambridge Univ. Press), 468

Peebles, P. J. E. 1980, The Large-Scale Structure of the Universe (Princeton: Princeton Univ. Press)

Peebles, P. J. E. 1984, ApJ, 284, 439

Peebles, P. J. E. 1993 Principles of Cosmology (Princeton: Princeton Univ. Press)

Perlmutter, S., Aldering, G., Goldhaber, G., et al. 1999, ApJ, 517,565

Podariu, S., \& Ratra, B. 2000, ApJ, 532, L109

Riess, A. G., Filippenko, A. V., Challis, P., et al. 1998, AJ, 116, 1009

Robertson, H. P. 1955, PASP, 67, 82

Sahni, V., \& Starobinsky, A. 2000, Int. J. Mod. Phys. D9, 373

Sandage, A. 1986, ApJ, 307, 1

Sandage, A. 1999, ApJ, 527, 479

Sandage, A., Tammann, G., \& Hardy, E. 1972, ApJ, 172, 253

Sylos Labini, F., Montuori, M., \& Pietronero, L. 1998, Phys. Rep., 293, 61

Teerikorpi, P. 1997, ARA\&A, 35, 101

Teerikorpi, P., Hanski, M., Theureau, G., et al. 1998, A\&A, 334, 395

Teerikorpi, P., \& Paturel, G. 2001, A\&A, submitted

Tikhonov, A., Makarov, D., \& Kopylov, A. 2000, Bull. Special Astrophys. Obs., 50, 39

van den Bergh, S. 1999, A\&A Rev., 9, 273

Wang, L., Caldwell, R. R., Ostriker, J. P., \& Steinhardt, P. J. 2000, ApJ, 530, 17

Wang, L., \& Steinhardt, P. J. 1998, ApJ, 508, 483

Weinberg, S. 1977, The First Three Minutes (Basic Books, New York), 26

Wetterich, C. 1995, A\&A, 301, 321

Wu, K. K. S., Lahav, O., \& Rees, M. J. 1999, Nature, 397, 225

Zeldovich, Ya. B. 1965, Advan. Astron. Ap., 3, 241 